\title{
A prospective, multicenter, observational study of long-term decitabine treatment in patients with myelodysplastic syndrome
}

\author{
Seong Hyun Jeong ${ }^{1}$, Yoo-Jin Kim², Je-Hwan Lee ${ }^{3}$, Yeo-Kyeoung Kim ${ }^{4}$, Soo Jeong \\ $\mathrm{Kim}^{5}$, Sung Kyu Park, Young Rok Do, Inho Kim ${ }^{8}$, Yeung-Chul Mun', Hoon Gu \\ $\mathrm{Kim}^{10}$, Won Sik Lee ${ }^{11}$, Hyeon Gyu Yi ${ }^{12}$, Young-Don Joo ${ }^{13}$, Chul Won Choi ${ }^{14}$, Suk Ran \\ $\mathrm{Kim}^{15}$, Sang Min $\mathrm{Na}^{15}$ and Jun Ho Jang ${ }^{16, *}$ \\ ${ }^{1}$ Department of Hematology-Oncology, Ajou University School of Medicine, Suwon, South Korea \\ 2 Department of Hematology, Seoul St. Mary's Hospital, College of Medicine, The Catholic University of Korea, Seoul, Korea \\ ${ }^{3}$ Department of Hematology, Asan Medical Center, Seoul, Korea \\ ${ }^{4}$ Department of Hematology, Chonnam National University Hwasun Hospital, Jeollanam-do, South Korea \\ ${ }^{5}$ Division of Hematology, Department of Internal Medicine, Severance Hospital, Yonsei University College of Medicine, Seoul, \\ Korea \\ ${ }^{6}$ Department of Internal Medicine, Soonchunhyang University Hospital, Bucheon, South Korea \\ 7 Division of Hematology-Oncology, School of Medicine, Keimyung University, Daegu, South Korea \\ ${ }^{8}$ Department of Internal Medicine, Seoul National University, Seoul, Korea \\ ${ }^{9}$ Department of Hematology and Oncology, School of Medicine, Ewha Womans University, Seoul, Korea \\ 10 Division of Hematology-Oncology, Department of Internal Medicine, Gyeongnam Regional Cancer Center, Institute of \\ Health Sciences, Gyeongsang National University School of Medicine, Jinju, South Korea \\ ${ }^{11}$ Department of Internal Medicine, Inje University College of Medicine, Inje University Busan Paik Hospital, Busan, South \\ Korea \\ 12 Department of Internal Medicine, Inha University College of Medicine, Incheon, South Korea \\ 13 Hematology-Oncology, Department of Internal Medicine, Inje University College of Medicine, Haeundae Paik Hospital, \\ Busan, South Korea \\ 14 Division of Oncology and Hematology, Department of Internal Medicine, Korea University Medical Center, Seoul, Korea \\ 15 Janssen Korea Medical Affairs, Seoul, Korea \\ 16 Division of Hematology and Oncology, Samsung Medical Center, Sungkyunkwan University School of Medicine, Seoul, \\ Korea \\ * For the Korean Society of Hematology AML/MDS Working Party
}

Correspondence to: Jun Ho Jang, email: junhojang@skku.edu

Keywords: decitabine, long-term treatment, myelodysplastic syndrome

Received: August 10, $2015 \quad$ Accepted: September 24, $2015 \quad$ Published: October 26, 2015

This is an open-access article distributed under the terms of the Creative Commons Attribution License, which permits unrestricted use, distribution, and reproduction in any medium, provided the original author and source are credited.

\section{ABSTRACT}

This prospective observational study evaluated the efficacy and safety of long-term decitabine treatment in patients with myelodysplastic syndrome (MDS). Decitabine $20 \mathrm{mg} / \mathrm{m}^{2} /$ day was administered intravenously for 5 consecutive days every 4 weeks to MDS patients in intermediate-1 or higher International Prognostic Scoring System (IPSS) risk categories. Active antimicrobial prophylaxis was given to prevent infectious complications. Overall response rate (ORR), overall survival (OS), progression-free survival (PFS), and time to response were evaluated, as were adverse events. The final analysis included 132 patients. IPSS risk was intermediate-2/high in $34.9 \%$ patients. The patients received a median of 5 cycles, with responders receiving a median of 8 cycles (range, 2-30). ORR was 62.9\% (complete response [CR], 36; partial response [PR], 3; marrow complete response [MCR], 19; and hematologic improvement, 25). Among responders, 39\% showed first response at cycle 3 or later. OS at 2 years was $60.9 \%$, with $17 \%$ progressing to acute myeloid leukemia. PFS at 


\section{2 years was $51.0 \%$. Patients achieving $\mathrm{mCR}$ showed comparable survival outcomes to those with CR/PR. With active antibiotic prophylaxis, febrile neutropenia events occurred in 61 of 1,033 (6\%) cycles. Long-term decitabine treatment with antibiotic prophylaxis showed favorable outcomes in MDS patients, and mCR predicted favorable survival outcomes.}

\section{INTRODUCTION}

Myelodysplastic syndrome (MDS) is a group of bone marrow disorders manifesting as cytopenias resulting from ineffective hematopoiesis and progressively evolving to acute myeloid leukemia (AML). Supportive treatment has long been the mainstay of treatment for a majority of patients, with allogeneic stem cell transplantation performed when available. DNA hypermethylation leading to inactivation of tumor-suppressor genes is the main pathobiologic mechanism in MDS [1-3], and the introduction of demethylating agents has changed the treatment paradigm of MDS.

Decitabine (5-aza-2'-deoxycytidine) reverses aberrant DNA hypermethylation of $\mathrm{CpG}$ islands by inhibiting DNA methyltransferase, resulting in reactivation of previously silenced tumor-suppressor genes [4]. Although decitabine as single-agent therapy failed to demonstrate overall survival (OS) benefit in contrast to azacitidine $[5,6]$, the United States Food and Drug Administration (US FDA) approved decitabine for the treatment of MDS, considering its comparable efficacy to azacitidine. Decitabine therapy has shown favorable treatment outcomes, with overall improvement rates of $42 \%-73 \%$ in previous studies [7-10]. Longer treatment duration may lead to better survival outcomes. A recent analysis from the AZA 001 trial showed that even patients with stable disease (SD) retained a survival benefit with azacitidine treatment and that late response could occur in one-third of patients with SD in earlier cycles [11]. Thus, continuation of treatment until disease progression is generally recommended. In previous studies, frequent, early treatment discontinuations, mainly due to febrile neutropenia and cytopenia-related infections, may have prevented patients from achieving the best clinical benefit of demethylating agents. A retrospective study showed a significant decrease in febrile episodes in patients receiving antibiotic prophylaxis [12]. We performed a multicenter, prospective observational study to determine whether long-term decitabine treatment with antibiotic prophylaxis and proper dose/schedule modification yielded better clinical outcomes in patients with MDS.

\section{PATIENTS AND METHODS}

\section{Patients}

The study included adult patients ( $\geq 20$ years) with MDS of any WHO subtype or chronic myelomonocytic leukemia, an IPSS score of 0.5 or more, and naïve to treatment with demethylating agents. Patients were excluded if they were allergic to decitabine, were pregnant or lactating, had progressed to AML ( $\geq 20 \%$ blasts), or had concurrent malignancy. Patients with active viral or bacterial infections were not included until complete recovery. This study was reviewed and approved by the institutional review board of each participating center, and all patients provided written informed consent. The study was registered at www.clinicaltrials.gov (NCT01400633).

\section{Treatments}

Decitabine was administered according to the 5-day intravenous outpatient schedule used in the ADOPT trial [8]. Patients received 5 consecutive injections of decitabine $20 \mathrm{mg} / \mathrm{m}^{2} /$ day over 1 hour every 4 weeks. Dose modification and delay of cycles were allowed per protocol guidelines when patients experienced severe adverse effects. Subsequent cycles could be delivered if the absolute neutrophil count was $>500 / \mathrm{mm}^{3}$ and platelet count was $>30,000 / \mathrm{mm}^{3}$. Treatment was delayed until recovery from non-hematological toxicities, such as serum creatinine $>2 \mathrm{mg} / \mathrm{dL}$, serum glutamate-pyruvate transaminase or total bilirubin $>2 \times$ upper normal limit, or active or uncontrolled infections. Active antibiotic prophylaxis was recommended to prevent infectious complications, especially in the first 4 cycles. Supportive measures, including antiemetics, transfusion, and growth factors, were allowed if needed. Continuation of decitabine treatment was recommended for at least 4 cycles, until progression or unacceptable AEs occurred.

\section{Study end points}

The primary end point of this study was the ORR, calculated as the sum of CR, PR, mCR, and HI. Secondary end points were OS, PFS, and time to response. Patients were followed-up for survival every 12 weeks until death. Response was evaluated by central review according to the modified IWG 2006 response criteria [14]. Treatment 
response was evaluated after completion of each cycle. Bone marrow biopsy was recommended at every 2 cycles, at the end of treatment, and when disease progression or recurrence was suspected.

\section{Safety evaluation}

Physical examination, complete blood count, and chemistry were performed at baseline and the first day of each cycle before initiation of treatment. The end of treatment evaluation was conducted $56 \pm 7$ days after the last dose of decitabine. AEs were evaluated based on the National Cancer Institute Common Terminology Criteria for Adverse Events (NCI CTCAE) ver. 4.0.

\section{Statistical analysis}

We analyzed the ORR, OS, PFS, and time to response in the FAS population, which comprised patients who met all of the inclusion/exclusion criteria, had a baseline assessment, received at least 2 cycles of decitabine treatment, and had at least one valid postbaseline clinical response assessment. ORR was defined as the proportion of patients with $\mathrm{CR}, \mathrm{mCR}, \mathrm{PR}$, and $\mathrm{HI}$ as the best response based on central review employing the IWG 2006 response criteria Confidence intervals for ORR were estimated with the Clopper-Pearson formula. OS was defined as the time from the start of decitabine treatment to death; data on survivors were censored at the last follow-up. PFS was defined as the time from the date of first decitabine administration to progression, relapse after showing $\mathrm{CR}$ or $\mathrm{PR}$, or death, whichever occurred first. Survival was calculated using the Kaplan-Meier method, and log-rank tests were used to compare the OS distribution by variables (best treatment response type and achievement of mCR). Time to first response and time to best response were analyzed with frequency and descriptive statistics (median, min-max) in each treatment cycle. Logistic regression analysis was used to assess the efficacy of decitabine post hoc within subgroups defined by the following baseline characteristics, which were determined to have effects on ORR: age $(\geq 65,<65)$, gender, period of MDS ( $\geq 1$ year, $<1$ year), WHO subtype, French-American-British (FAB) subtype, IPSS category, and karyotype. Safety was analyzed in all patients who received at least one dose of decitabine. All $P$-values were two-sided and statistical significance was accepted at the level of $P<0.05$.

\section{RESULTS}

\section{Patient characteristics}

The study was conducted at 33 centers in Korea from December 2010 to October 2011. Of the 158 patients enrolled, 2 patients were excluded from the safety analysis for non-initiation of the study drug (decitabine), and 24 patients were excluded from the full analysis set (FAS) for not meeting inclusion criteria (3 patients), dropout before 2 cycles ( 5 patients), and not undergoing response evaluation (16 patients). Thus, 132 patients were included in the FAS (Figure 1). Their median age was 63 years (range, 20-82 years), and a majority of the patients were

\begin{tabular}{|c|c|c|c|}
\hline Baseline & $\mathrm{N}=158$ & & \\
\hline & & Exclusion & $\mathrm{n}=\mathbf{2}$ \\
\hline & & No initiation of study drug & $\mathbf{n}=\mathbf{2}$ \\
\hline \multirow[t]{3}{*}{ Safety set } & $n=156$ & & \\
\hline & & Exclusion & $n=24$ \\
\hline & & $\begin{array}{l}\text { Unmet inclusion criteria } \\
\text { No response evaluation } \\
\text { Less than } 2 \text { cycles }\end{array}$ & $\begin{array}{r}n=3 \\
n=16 \\
n=5\end{array}$ \\
\hline \multirow[t]{7}{*}{ Full Analysis Set } & $\mathbf{n}=\mathbf{1 3 2}$ & & \\
\hline & & Exclusion & $\mathbf{n}=\mathbf{3 0}$ \\
\hline & & Study end & $\mathrm{n}=7$ \\
\hline & & $\begin{array}{l}\text { Reason for treatment } \\
\text { discontinuation }\end{array}$ & $\mathbf{n}=\mathbf{2 3}$ \\
\hline & & Consent withdrawal & $n=14$ \\
\hline & & Follow-up loss & $n=4$ \\
\hline & & Other & $\mathbf{n}=\mathbf{5}$ \\
\hline
\end{tabular}

\section{Figure 1: Patient flowchart}


Table 1: Patient characteristics $(\mathrm{N}=132)$

\begin{tabular}{|c|c|c|}
\hline \multirow{2}{*}{$\begin{array}{l}\text { Characteristics } \\
\text { Gender }\end{array}$} & \multicolumn{2}{|c|}{ n (\%) } \\
\hline & & \\
\hline Male & 76 & $(57.58 \%)$ \\
\hline Female & 56 & $(42.42 \%)$ \\
\hline \multicolumn{3}{|l|}{ Age (years) } \\
\hline Median (range) & 63 & $(20-82)$ \\
\hline \multicolumn{3}{|c|}{ Duration of disease (days) } \\
\hline Median (range) & 19 & $(1-3,011)$ \\
\hline \multicolumn{3}{|l|}{ Comorbidity } \\
\hline Yes & 38 & $(28.79 \%)$ \\
\hline No & 94 & $(71.21 \%)$ \\
\hline \multicolumn{3}{|l|}{ MDS type } \\
\hline De novo & 127 & $(96.21 \%)$ \\
\hline Secondary & 5 & $(3.79 \%)$ \\
\hline \multicolumn{3}{|l|}{ WHO subtype } \\
\hline RCUD & 4 & $(3.03 \%)$ \\
\hline RARS & 3 & $(2.27 \%)$ \\
\hline RCMD & 40 & $(30.30 \%)$ \\
\hline RAEB-1 & 30 & $(22.73 \%)$ \\
\hline RAEB-2 & 36 & $(27.27 \%)$ \\
\hline MDS-U & 6 & $(4.55 \%)$ \\
\hline $\operatorname{Del}(5 q)$ & 1 & $(0.76 \%)$ \\
\hline CMML-1 & 7 & $(5.30 \%)$ \\
\hline CMML-2 & 4 & $(3.03 \%)$ \\
\hline Unclassified & 1 & $(0.76 \%)$ \\
\hline \multicolumn{3}{|l|}{ IPSS risk category } \\
\hline Intermediate-1 & 86 & $(65.15 \%)$ \\
\hline Intermediate- 2 & 36 & $(27.27 \%)$ \\
\hline High & 10 & $(7.58 \%)$ \\
\hline \multicolumn{3}{|c|}{ ECOG performance status } \\
\hline 0,1 & 109 & $(82.58 \%)$ \\
\hline$\geq 2$ & 23 & $(17.42 \%)$ \\
\hline \multicolumn{3}{|c|}{ Karyotype at baseline } \\
\hline Good & 75 & $(56.82 \%)$ \\
\hline Normal & 69 & $(52.27 \%)$ \\
\hline Other & 6 & $(4.55 \%)$ \\
\hline Intermediate & 27 & $(20.45 \%)$ \\
\hline Poor & 22 & $(16.67 \%)$ \\
\hline Not done & 8 & $(6.06 \%)$ \\
\hline
\end{tabular}

CMML, chronic myelomonocytic leukemia; Del, deletion; ECOG, Eastern Cooperative Oncology Group; IPSS, International Prognostic Scoring System; MDS, myelodysplastic syndrome; MDS-U, unclassifiable MDS; RAEB, refractory anemia with excess of blasts; RARS, refractory anemia with ringed sideroblasts; RCMD, refractory cytopenia with multilineage dysplasia; RCUD, refractory cytopenia with unilineage dysplasia; WHO, World Health Organization

male (57.6\%). The median duration of disease was 19 days (range, 1-3,011 days). Patients were categorized according to the International Prognostic Scoring System (IPSS) into intermediate-1 (Int-1; 65\%), intermediate-2 (Int-2; 27\%), and high $(7.6 \%)$ risk groups. Five patients had secondary MDS. At the baseline evaluation, karyotype was good in 75 patients $(57 \%)$, intermediate in $27(20 \%)$, and poor in $22(17 \%)$; karyotyping data was unavailable in 8 patients.
Only 3 patients had previously received other treatments before decitabine treatment (androgen, 2; erythropoietin, 1). Patient characteristics are summarized in Table 1.

\section{Treatment exposure and response}

A total of 1,033 cycles were delivered to 132 patients (median, 5 cycles), with $84 \%$ patients receiving 


\begin{tabular}{|c|c|c|}
\hline \multirow{2}{*}{$\begin{array}{l}\text { Response } \\
\text { CR }\end{array}$} & \multicolumn{2}{|c|}{ n $(\%)$} \\
\hline & 36 & $(27.27 \%)$ \\
\hline PR & 3 & $(2.27 \%)$ \\
\hline mCR with HI & 8 & $(6.06 \%)$ \\
\hline mCR without HI & 11 & $(8.33 \%)$ \\
\hline HI only* & 25 & $(18.94 \%)$ \\
\hline Patient experienced HI & 72 & \\
\hline $\mathrm{SD}^{\#}$ & 48 & $(36.36 \%)$ \\
\hline $\mathrm{ORR}(\mathrm{CR}+\mathrm{PR}+\mathrm{mCR}+\mathrm{HI})$ & 83 & $(62.88 \%)$ \\
\hline $95 \% \mathrm{CI}^{+}$ & $(54.04 \%$ & $-71.12 \%)$ \\
\hline $\mathrm{CR}+\mathrm{PR}+\mathrm{mCR}+\mathrm{HI}+\mathrm{SD}$ & 131 & $(99.24 \%)$ \\
\hline Failure & 1 & $(0.76 \%)$ \\
\hline Cytogenetic response & $55^{* *}$ & \\
\hline $\mathrm{CR}$ & 14 & $(25.45 \%)$ \\
\hline PR & 5 & $(9.09 \%)$ \\
\hline NE/ND & 36 & $(65.45 \%)$ \\
\hline \multicolumn{3}{|c|}{$\begin{array}{l}\text { CR, complete response; HI, hematologic improvement; HI-E, erythroid response; HI-N, } \\
\text { neutrophil response; HI-P, platelet response; IWG, International working group; mCR, } \\
\text { marrow complete response; ND, not done; NE, not evaluable; ORR, overall response rate; } \\
\text { PR, partial response; SD, stable disease } \\
\text { *HI only: HI in SD and NE/ND } \\
\text { \#SD excluding other than HI } \\
{ }^{+} 95 \% \text { confidence interval for proportion of ORR } \\
\text { ** In case of abnormal Karyotype (Good-Other, Intermediate, Poor) }\end{array}$} \\
\hline
\end{tabular}

3 or more cycles and $47 \%$ receiving 6 or more cycles. The median number of cycles among responders was 8 (range, 2-30 cycles). The mean interval between cycles was 36.8 \pm 9.4 days. Dose modification was required in 8 patients. At the time of analysis, 7 patients remained adherent to the treatment. Reasons for treatment discontinuation included failure to achieve response (11\%), progression $(8 \%)$, adverse events (AEs; 7\%), proceeding to hematopoietic stem cell transplantation (HSCT; 26\%), progression to AML (13\%), progression after achieving response (5\%), patient decision $(11 \%)$, death (12\%), and loss to followup loss or other reasons (7\%). Prophylactic antimicrobial agents were administered to $86 \%$ patients, including antibacterials (aminopenicillin or quinolone) to $83.3 \%$, antifungals (fluconazole or itraconazole) to $77 \%$, and antivirals (acyclovir) to $28 \%$.

The overall response rate (ORR) was $62.9 \%$ (95\% CI, 54.0\%-71.1\%): complete response (CR), 27.3\%; partial response (PR), 2.3\%; marrow complete response (mCR), $14.4 \%$; and hematologic improvement $(\mathrm{HI}), 18.9 \%$. Initial response was achieved within 2 cycles in $61 \%$ patients; however, $39 \%$ patients showed initial response at cycle 3 or later (31\% at cycles 3 and 4; Figure 2A). The best response peaked at cycle 4 (28\%; Figure $2 \mathrm{~B})$. HI was observed in $44.4 \%$ patients (erythroid response [HI-E],
26.2\%; platelet response [HI-P], 31\%; and neutrophil response [HI-N], 16.7\%). The median number of cycles to HI was 2 (range, 1-13). Cytogenetic response was noted in 19 of 55 patients (34.6\%; CR, 25.5\% and PR, 9.1\%) with abnormalities at baseline. Progression to AML was $10.8 \%$ at 1 year and $17 \%$ at 2 years. Karyotype at diagnosis was a significant prognostic factor for ORR (Table 3). Patients with good or poor karyotype showed better ORR than those with an intermediate type.

\section{Survival data}

The median follow-up duration was 9.5 months. Among 132 patients in FAS, fifty-nine (44.7\%) patients died during the treatment $(16.7 \%)$ or follow-up period (28\%). Causes of death were disease progression (24\%), AEs $(20 \%)$, and other reasons $(56 \%)$. Other reasons of death included pneumonia in 10 patients and sepsis/septic shock in 6 patients.

The 1-year and 2-year OS was $80.9 \%(95 \% \mathrm{CI}$, $72.9 \%-86.8 \%$ ) and $60.9 \%$ (95\% CI, $51.3 \%-69.1 \%$ ), respectively. The progression free survival (PFS) rate was $70.0 \%(95 \% \mathrm{CI}, 61.1 \%-77.2 \%)$ at 1 year and $51 \%$ (95\% CI, $41.5 \%-59.6 \%)$ at 2 years. Patients who achieved 
Table 3: Prognostic factor analysis for overall response

\begin{tabular}{|c|c|c|c|c|c|c|c|}
\hline \multirow{2}{*}{ Characteristic } & \multicolumn{2}{|c|}{ Response } & \multirow[t]{2}{*}{$P$-value: } & \multirow{2}{*}{ Odds ratio } & \multicolumn{2}{|c|}{$95 \% \mathrm{CI}$} & \multirow{2}{*}{$P$-value* } \\
\hline & $\mathbf{n} / \mathbf{N}$ & $(\%)$ & & & Lower & Upper & \\
\hline \multicolumn{8}{|l|}{ Age, years } \\
\hline$\leq 65$ & $46 / 72$ & $(63.89 \%)$ & 0.7924 & 1 & & & 0.7925 \\
\hline$>65$ & $37 / 60$ & $(61.67 \%)$ & & 0.909 & 0.448 & 1.847 & \\
\hline \multicolumn{8}{|l|}{ Sex } \\
\hline Male & $47 / 76$ & $(61.84 \%)$ & 0.774 & 1 & & & 0.7740 \\
\hline Female & $36 / 56$ & $(64.29 \%)$ & & 1.111 & 0.543 & 2.273 & \\
\hline \multicolumn{8}{|l|}{ Duration of MDS } \\
\hline$\leq 1$ year & $76 / 116$ & $(65.52 \%)$ & 0.0911 & 1 & & & 0.0985 \\
\hline$>1$ year & $7 / 16$ & $(43.75 \%)$ & & 0.409 & 0.142 & 1.181 & \\
\hline \multicolumn{8}{|l|}{ WHO subtype } \\
\hline RCUD & $1 / 4$ & $(25.00 \%)$ & 0.2 & 1 & & & \\
\hline RARS & $2 / 3$ & $(66.67 \%)$ & & 6.000 & 0.221 & 162.531 & \\
\hline RCMD & $22 / 40$ & $(55.00 \%)$ & & 3.667 & 0.351 & 38.345 & \\
\hline RAEB-1 & $23 / 30$ & $(76.67 \%)$ & & 9.857 & 0.880 & 110.425 & \\
\hline RAEB-2 & $26 / 36$ & $(72.22 \%)$ & & 7.800 & 0.723 & 84.091 & 0.3437 \\
\hline MDS-U & $2 / 6$ & $(33.33 \%)$ & & 1.500 & 0.089 & 25.392 & \\
\hline $\operatorname{Del}(5 q)$ & $1 / 1$ & $(100.00 \%)$ & & $>999.999$ & $<0.001$ & $>999.999$ & \\
\hline CMML-1 & $3 / 7$ & $(42.86 \%)$ & & 2.250 & 0.149 & 33.933 & \\
\hline CMML-2 & $2 / 2$ & $(50.00 \%)$ & & 3.000 & 0.150 & 59.890 & \\
\hline Unclassified & $1 / 1$ & $(100.00 \%)$ & & $>999.999$ & $<0.001$ & $>999.999$ & \\
\hline \multicolumn{8}{|l|}{ IPSS risk category } \\
\hline Intermediate-1 & $53 / 86$ & $(61.63 \%)$ & 0.5062 & 1 & & & \\
\hline Intermediate-2 & $22 / 36$ & $(61.11 \%)$ & & 0.978 & 0.440 & 2.175 & 0.5265 \\
\hline High & $8 / 10$ & $(80.00 \%)$ & & 2.491 & 0.498 & 12.451 & \\
\hline Karyotype at baseline & & & & & & & 0.0239 \\
\hline Good (normal or other) & $53 / 75$ & $(70.67 \%)$ & 0.0191 & 1 & & & \\
\hline Intermediate & $11 / 27$ & $(40.74 \%)$ & & 0.285 & 0.114 & 0.712 & $0.0112 * *$ \\
\hline Poor & $15 / 22$ & $(68.18 \%)$ & & 0.890 & 0.319 & 2.481 & $0.3211^{* *}$ \\
\hline
\end{tabular}

CMML, chronic myelomonocytic leukemia; CR, complete response; Del, deletion; HI, hematologic improvement; IPSS, International Prognostic Scoring System; mCR, marrow complete response; MDS, myelodysplastic syndrome; MDS-U, unclassifiable MDS; ORR, overall response rate; PR, partial response; RAEB, refractory anemia with excess of blasts; RARS, refractory anemia with ringed sideroblasts; RCMD, refractory cytopenia with multilineage dysplasia; RCUD, refractory cytopenia with unilineage dysplasia

$* P$-value is obtained by logistic regression (Wald test - testing global null hypothesis)

$* * P$-value is obtained by logistic regression

$\$ P$-value is obtained by Chi-square test

response with decitabine treatment (responders) showed better OS at 1 year $(88.8 \%$ vs. $66.7 \%, P=0.0035)$ and 2 years $(70.6 \%$ vs. $42.5 \%, P=0.0014)$ than those who did not achieve response (nonresponders; Figure 3B). Patients who achieved $\mathrm{mCR}$ showed comparable survival with those achieved CR or PR, regardless of HI (Figure 3C). Patients who achieved HI showed better survival than those who did not. This was statistically significant in the higher risk IPSS groups (INT-2 and high), but not in the lower risk group.

\section{Adverse events}

The safety analysis included 156 patients. Febrile neutropenia was the most common $\mathrm{AE}$, considered to be possibly related to the study drug in $23.7 \%$ patients. Febrile neutropenia events occurred in 61 of 1,033 (6\%) cycles in 37 patients in the FAS. Grade III or more AEs included neutropenia (21.2\%), anemia (12.8\%), thrombocytopenia (15.4\%), and febrile neutropenia (23.7\%). Non-hematologic AEs occurring in $>10 \%$ patients included nausea (27.6\%), decreased appetite 
(23.7\%), and pyrexia (23.1\%). Documented infection included upper respiratory tract infection in 5 patients, pneumonia in 6 , sepsis in 5 , and cellulitis in 2. Grade III or IV non-hematologic AEs included nausea (1.3\%), decreased appetite (3.2\%), and pyrexia (3.9\%).

\section{DISCUSSION}

This is one of the largest multicenter studies prospectively evaluating the efficacy of decitabine in patients with MDS. In previous studies, the primary cause of early discontinuation of decitabine treatment, other than treatment failure, was cytopenia-related infections. Therefore, to prevent early dropout, all patients in this study were to receive antibacterial prophylaxis as well as dose and schedule modification if they experienced cytopenias at the first cycle. This led to a reduction in febrile neutropenia events to $6 \%$ (62 of 1,033 cycles) in contrast to $11 \%-14 \%$ reported with the same treatment schedule in previous studies $[8,9]$. This translated into a decrease in early discontinuation rates $(84 \%$ patients

A.

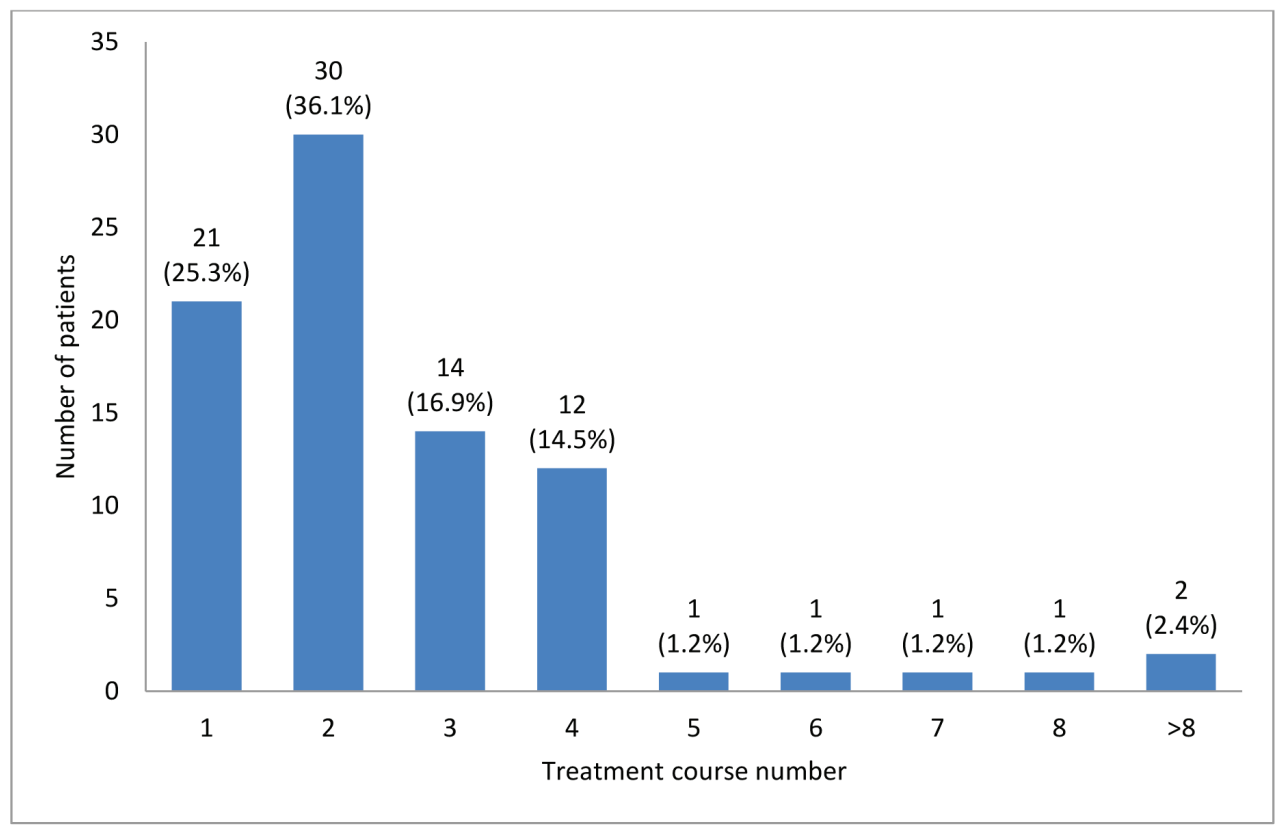

B.

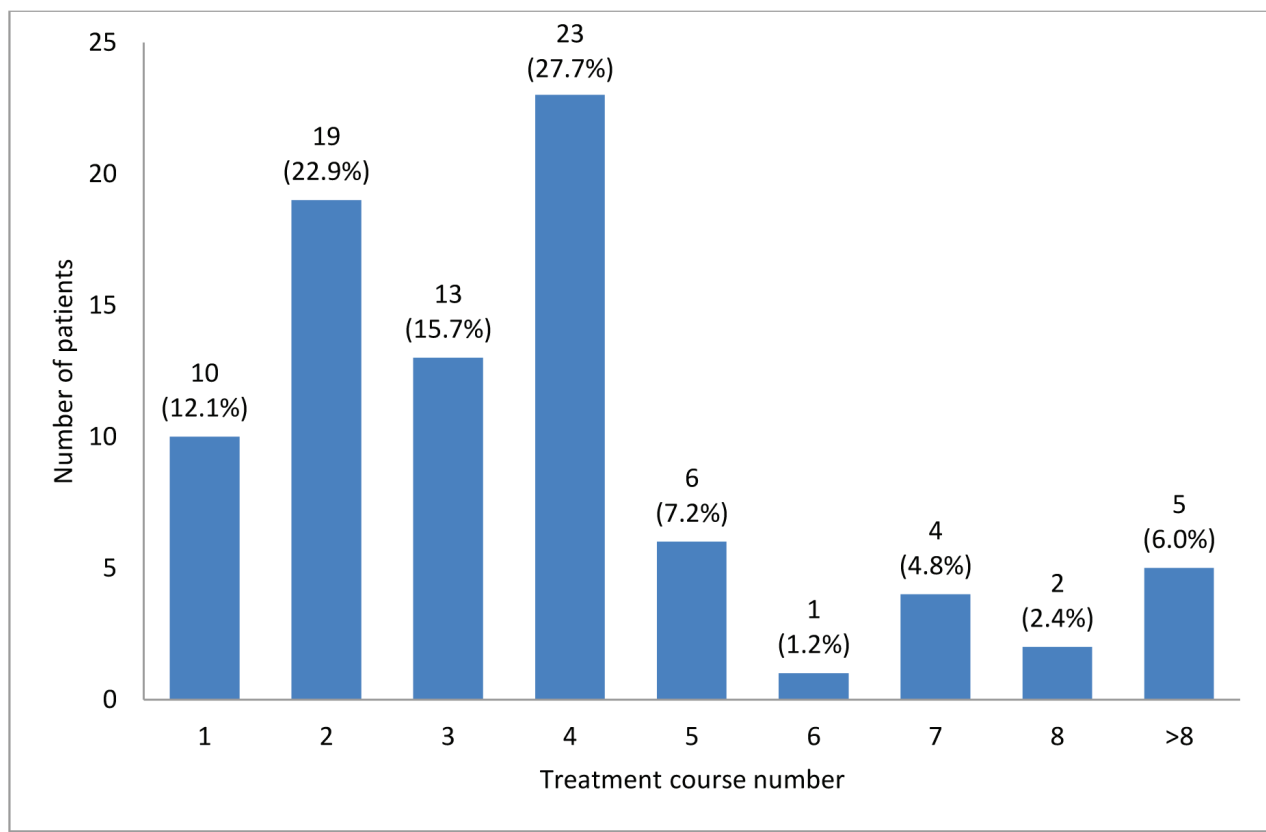

Figure 2: Time to first response A. and time to best response B. 


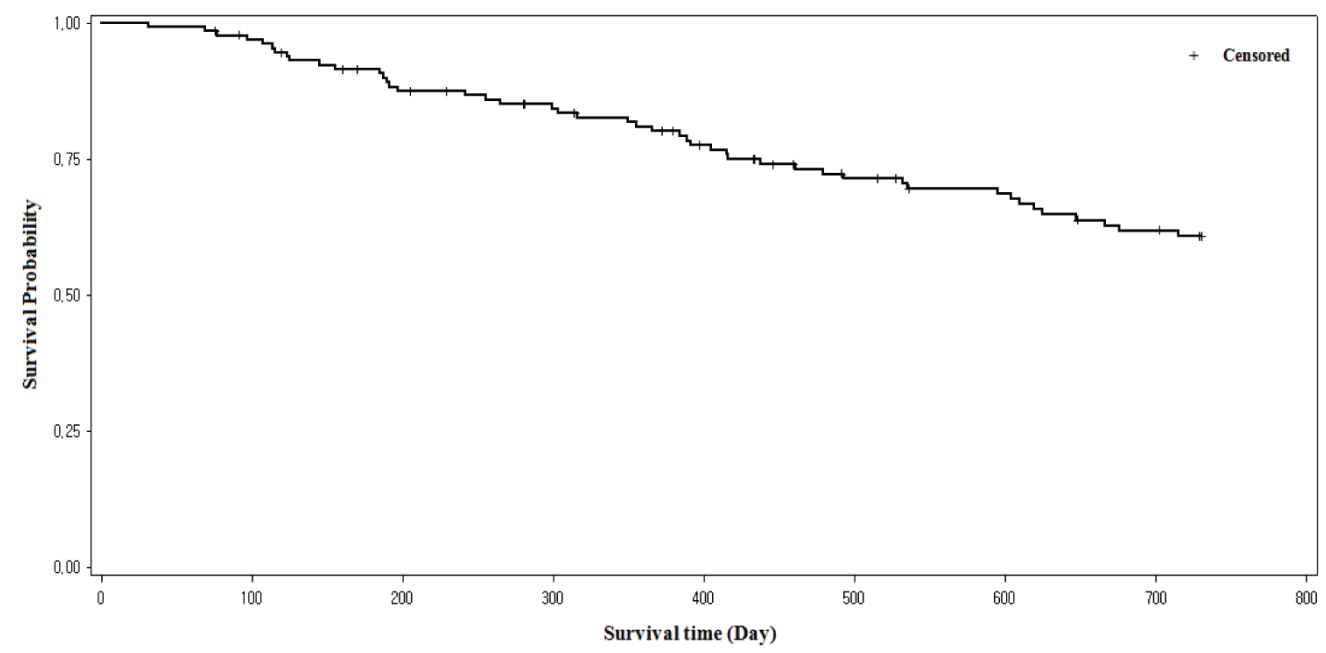

B.

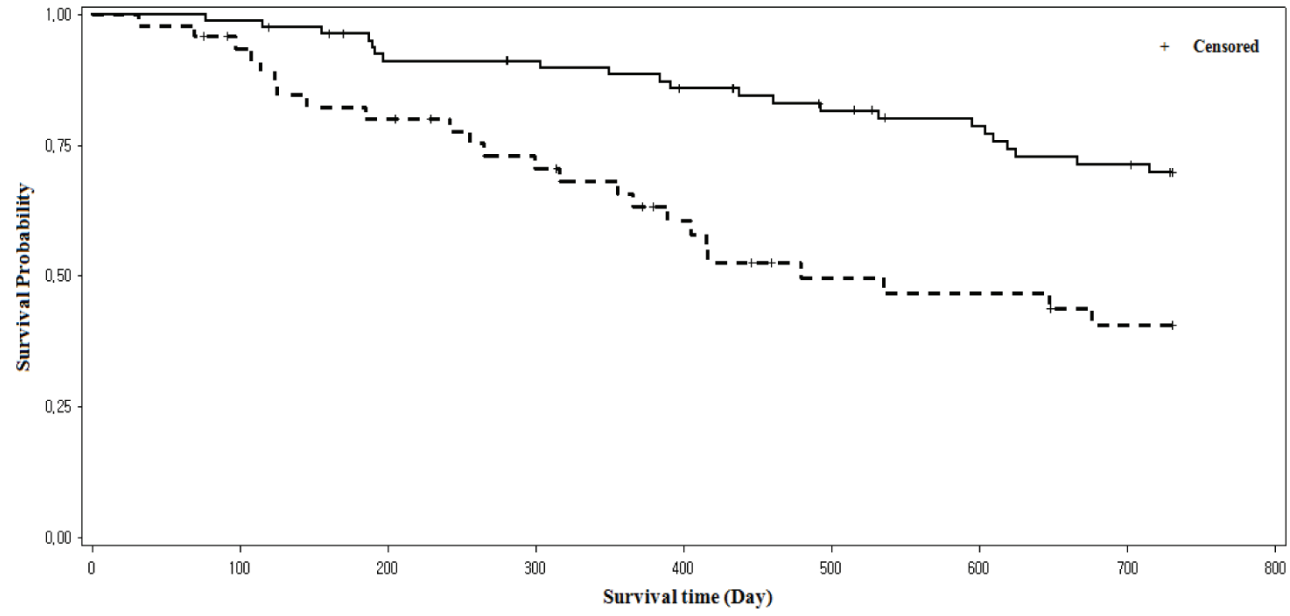

C.

Response $\_$responder $\quad . . .$. non-responder

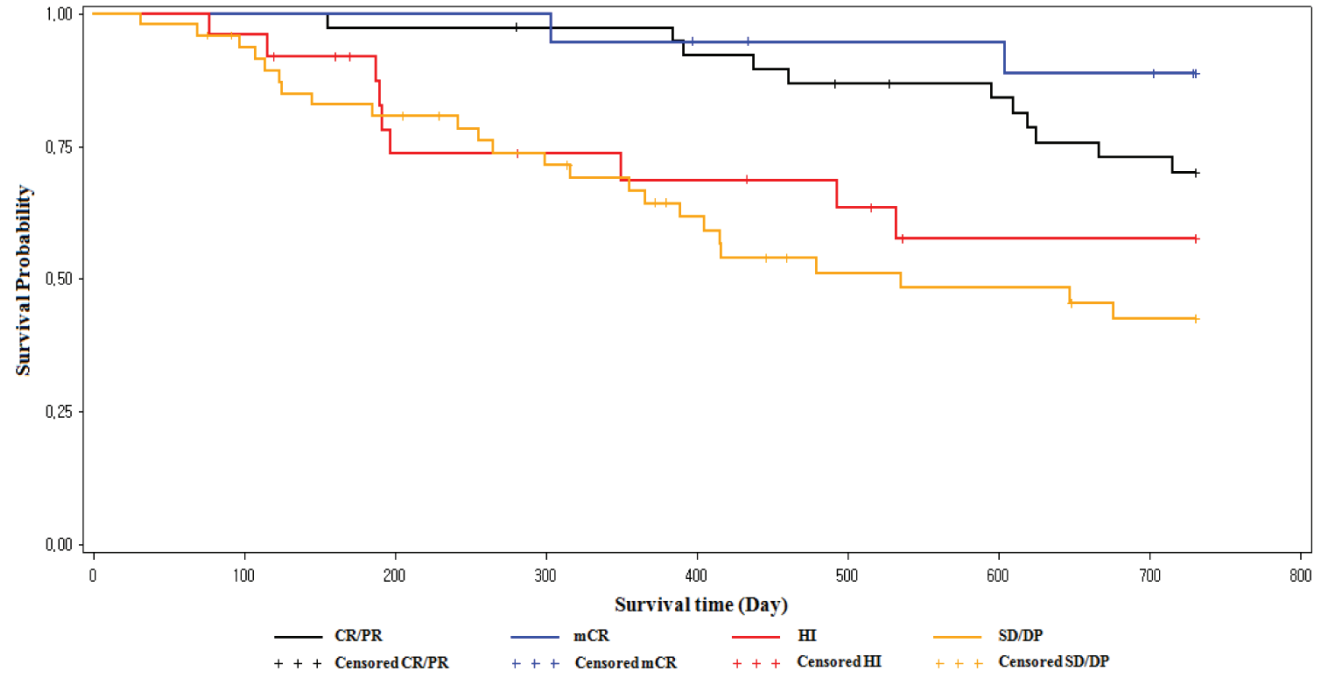

Figure 3: Overall survival (OS; A.), OS in responders vs. non-responders B., and OS by response type $\mathbf{C}$. 
received at least 3 cycles) and increase in the median number of cycles.

Overall treatment outcomes in this study compare favorably with those from previous studies using the 5-day outpatient protocol [8-10]. The CR and ORR in this study were somewhat higher than those in the DIVA study [9]. This could be attributed mainly to the patient characteristics, with our study including more IPSS Int-1 patients than previous studies. Furthermore, most patients were newly diagnosed and were treatment naive. Only 5 patients with secondary MDS were included. In addition, $47 \%$ patients received at least 6 cycles, with the responders receiving a median of 8 cycles. Baseline cytogenetic data was available for $94 \%$ patients. Of the 55 patients with cytogenetic abnormalities at baseline, 19 patients (35\%) showed cytogenetic response (14 CR and $5 \mathrm{PR}$ ). Interestingly, the response rate to decitabine was inferior in patients with an intermediate karyotype as compared to patients with good or poor risk cytogenetics. Thus, decitabine may have better effects on clones with more cytogenetic/genetic alterations.

The clinical significance of $\mathrm{mCR}$ remains unclear. In previous studies, $15 \%-23 \%$ decitabine-treated patients showed mCR $[5,9,10]$. The $14 \%$ patients who achieved mCR in our study showed comparable survival outcomes with the $\mathrm{CR} / \mathrm{PR}$ patients regardless of $\mathrm{HI}$ status. $\mathrm{mCR}$ seems to be an analogue of CR with incomplete blood count recovery (CRi) in AML, reflecting a low tumor burden but with decreased marrow function. Because normal hematopoiesis is suppressed in MDS, it is natural that a substantial proportion of patients show $\mathrm{mCR}$ after successful treatment with demethylating agents. In a retrospective analysis, patients with $\mathrm{mCR}$ showed comparable outcomes to those with CR after HSCT [13]. In the present study, patients who achieved mCR showed favorable survival outcomes regardless of HSCT status. However, this finding is discordant with a previous study, where patients achieving $\mathrm{mCR}$ showed inferior survival outcomes compared to those with CR or PR [9]. Further studies are required to clarify this effect.

HI was a significant predictor of OS in the DIVA study [9]. This finding was confirmed in our study. As in the DIVA study [9], HI was associated with better survival in higher risk patients (IPSS INT-2 or high), but not in lower risk patients (INT-1). Recently, an analysis of higher risk patients treated with azacitidine showed that patients who achieved $\mathrm{HI}$ without response (CR or PR) also experienced a survival benefit (hazard ratio $0.19 ; 95 \% \mathrm{CI}$, $0.08-0.46 ; P<0.001$ ) [11]. Thus, HI could be accepted as a predictor of better survival in higher risk MDS patients treated with demethylating agents.

In previous studies, response to demethylating agents was observed early in the course of treatment, usually within 2 cycles $[5,6,9,10]$. In our study, however, a higher proportion of patients (37.6\%) showed first response only at cycle 3 or later. We do not have a clear explanation for this observation. However, the median cycle duration was 35 days in our study, which is significantly longer than in previous studies. The lower early dose intensity in our study than in the other studies may explain the difference. However, the ORR in our study was not inferior to those in other studies. As shown in previous studies, the number of treatment cycles is a significant factor determining the effect of demethylating agents $[5,6,10]$. Thus, prolonged administration of decitabine is required for the treatment of MDS. In addition, considering that a significant number of patients respond to decitabine only after 3 or more cycles, effectiveness of decitabine therapy should be assessed after completion of at least 4 cycles, especially in patients showing SD in earlier cycles. This finding was supported by a recent analysis of the AZA 001 trial [11].

The major difference between the AZA-001 and GMDSSG/EORTC 06011 trials was the median number of treatment cycles ( 9 vs. 4) $[5,6]$. In addition, the M. D. Anderson Cancer Center study comparing 3 decitabine schedules showed that a high dose-intensity schedule induces more favorable responses [10]. To maximize the treatment outcomes of demethylating agents, both dose-intensity and treatment cycles are important. Thus, prevention of early drop-out and continuation of decitabine treatment until failure is recommended.

In conclusion, long-term decitabine treatment was effective for Int-1 or higher risk patients with MDS, with an acceptable toxicity profile. Appropriate antibacterial prophylaxis was effective in reducing early febrile neutropenia events. Patients with $\mathrm{mCR}$ showed comparable survival to those with $\mathrm{CR} / \mathrm{PR}$, and $\mathrm{mCR}$ can be considered a favorable response to decitabine treatment.

\section{ACKNOWLEDGMENTS}

This research was supported by Janssen Korea.

\section{CONFLICTS OF INTEREST}

There is no conflict of interest.

\section{REFERENCES}

1. Steensma DP and Tefferi A. The myelodysplastic syndrome(s): a perspective and review highlighting current controversies. Leukemia research. 2003; 27: 95-120.

2. Santini V, Kantarjian HM and Issa JP. Changes in DNA methylation in neoplasia: pathophysiology and therapeutic implications. Annals of internal medicine. 2001; 134: 573586.

3. Leone G, Teofili L, Voso MT and Lubbert M. DNA methylation and demethylating drugs in myelodysplastic syndromes and secondary leukemias. Haematologica. 2002; 87: 1324-1341. 
4. Jones PA and Taylor SM. Cellular differentiation, cytidine analogs and DNA methylation. Cell. 1980; 20: 85-93.

5. Lubbert M, Suciu S, Baila L, Ruter BH, Platzbecker U, Giagounidis A, Selleslag D, Labar B, Germing U, Salih HR, Beeldens F, Muus P, Pfluger KH, et al. Low-dose decitabine versus best supportive care in elderly patients with intermediate- or high-risk myelodysplastic syndrome (MDS) ineligible for intensive chemotherapy: final results of the randomized phase III study of the European Organisation for Research and Treatment of Cancer Leukemia Group and the German MDS Study Group. Journal of clinical oncology. 2011; 29: 1987-1996.

6. Fenaux P, Mufti GJ, Hellstrom-Lindberg E, Santini V, Finelli C, Giagounidis A, Schoch R, Gattermann N, Sanz G, List A, Gore SD, Seymour JF, Bennett JM, et al. Efficacy of azacitidine compared with that of conventional care regimens in the treatment of higher-risk myelodysplastic syndromes: a randomised, open-label, phase III study. The Lancet Oncology. 2009; 10: 223-232.

7. Kantarjian H, Issa JP, Rosenfeld CS, Bennett JM, Albitar M, DiPersio J, Klimek V, Slack J, de Castro C, Ravandi F, Helmer R, 3rd, Shen L, Nimer SD, et al. Decitabine improves patient outcomes in myelodysplastic syndromes: results of a phase III randomized study. Cancer. 2006; 106: 1794-1803.

8. Steensma DP, Baer MR, Slack JL, Buckstein R, Godley LA, Garcia-Manero G, Albitar M, Larsen JS, Arora S, Cullen MT and Kantarjian H. Multicenter study of decitabine administered daily for 5 days every 4 weeks to adults with myelodysplastic syndromes: the alternative dosing for outpatient treatment (ADOPT) trial. Journal of clinical oncology. 2009; 27: 3842-3848.

9. Lee JH, Jang JH, Park J, Park S, Joo YD, Kim YK, Kim HG, Choi CW, Kim SH, Park SK, Park E and Min YH. A prospective multicenter observational study of decitabine treatment in Korean patients with myelodysplastic syndrome. Haematologica. 2011; 96: 1441-1447.
10. Kantarjian H, Oki Y, Garcia-Manero G, Huang X, O’Brien S, Cortes J, Faderl S, Bueso-Ramos C, Ravandi F, Estrov Z, Ferrajoli A, Wierda W, Shan J, et al. Results of a randomized study of 3 schedules of low-dose decitabine in higher-risk myelodysplastic syndrome and chronic myelomonocytic leukemia. Blood. 2007; 109: 52-57.

11. Gore SD, Fenaux P, Santini V, Bennett JM, Silverman LR, Seymour JF, Hellstrom-Lindberg E, Swern AS, Beach CL and List AF. A multivariate analysis of the relationship between response and survival among patients with higherrisk myelodysplastic syndromes treated within azacitidine or conventional care regimens in the randomized AZA-001 trial. Haematologica. 2013; 98: 1067-1072.

12. Lee JH, Lee KH, Lee JH, Kim DY, Kim SH, Lim SN, Kim SD, Choi Y, Lee SM, Lee WS, Choi MY and Joo YD. Decreased incidence of febrile episodes with antibiotic prophylaxis in the treatment of decitabine for myelodysplastic syndrome. Leukemia research. 2011; 35: 499-503.

13. Kim DY, Lee JH, Park YH, Lee JH, Kim SD, Choi Y, Lee SB, Lee KH, Ahn SY, Lee YS, Seol M, Kang YA, Jeon $\mathrm{M}$, et al. Feasibility of hypomethylating agents followed by allogeneic hematopoietic cell transplantation in patients with myelodysplastic syndrome. Bone marrow transplantation. 2012; 47: 374-379.

14. Cheson BD, Greenberg PL, Bennett JM, Lowenberg B, Wijermans PW, Nimer SD, Pinto A, Beran M, de Witte TM, Stone RM, Mittelman M, Sanz GF, Gore SD, et al. Clinical application and proposal for modification of the International Working Group (IWG) response criteria in myelodysplasia. Blood. 2006; 108: 419-425. 